\title{
Electroweak monopoles and magnetic dumbbells in grand unified theories
}

\author{
G. Lazarides $\oplus^{1, *}$ and Q. Shafi ${ }^{2, \dagger}$ \\ ${ }^{1}$ School of Electrical and Computer Engineering, Faculty of Engineering, \\ Aristotle University of Thessaloniki, Thessaloniki 54124, Greece \\ ${ }^{2}$ Bartol Research Institute, Department of Physics and Astronomy, University of Delaware, \\ Newark, Delaware 19716, USA
}

(Received 18 February 2021; accepted 15 April 2021; published 20 May 2021)

\begin{abstract}
We use the $S U(5)$ model to show the presence in grand unified theories of an electroweak monopole and a magnetic dumbbell ("meson") made up of a monopole-antimonopole pair connected by a Z-magnetic flux tube. The monopole is associated with the spontaneous breaking of the weak $S U(2)_{L}$ gauge symmetry by the induced vacuum expectation value of a heavy scalar $S U(2)_{L}$ triplet with zero weak hypercharge contained in the adjoint Higgs 24-plet. This monopole carries a Coulomb magnetic charge of $(3 / 4)(2 \pi / e)$ as well as $Z$-magnetic charge, where $2 \pi / e$ denotes the unit Dirac magnetic charge. Its total magnetic charge is $\sqrt{3 / 8}(4 \pi / e)$, which is in agreement with the Dirac quantization condition. The monopole weighs about $700 \mathrm{GeV}$, but because of the attached $Z$-magnetic tube it exists, together with the antimonopole, in a magnetic dumbbell configuration whose mass is expected to lie in the $\mathrm{TeV}$ range. The presence of these topological structures in $S U(5)$ and $S O(10)$ and in their supersymmetric extensions provides an exciting new avenue for testing these theories in high-energy colliders.
\end{abstract}

DOI: 10.1103/PhysRevD.103.095021

Grand unified theories based on the gauge groups $S U(5)$ [1], $S O(10)$ [2], and $E_{6}$ [3] all predict the existence of a superheavy and topologically stable magnetic monopole, which carries a single quantum $(2 \pi / e)$ of Dirac magnetic charge [4] as well as color magnetic charge [5,6]. In $S U(5)$ this is the lightest monopole, but $S O(10)$ and $E_{6}$ may also give rise to topologically stable intermediate-scale monopoles, depending on their symmetry-breaking patterns. For instance, $S O(10)$ breaking via $S U(4)_{c} \times S U(2)_{L} \times S U(2)_{R}$ [7] yields an intermediate-scale topologically stable monopole carrying two units of the Dirac charge as well as color magnetic charge [8]. Depending on its symmetry-breaking scale, the trinification symmetry group $S U(3)_{c} \times S U(3)_{L} \times$ $S U(3)_{R}$ can yield a topologically stable triply charged monopole with mass in the $\mathrm{TeV}$ region, thus making it potentially accessible at high-energy colliders [9].

In this paper, we display the presence of another class of topological structures that can appear in grand unified theories. These structures are not topologically stable, and, as we show using the $S U(5)$ example, they are magnetic dumbbells (or "mesons") made up of electroweak

\footnotetext{
glazarid@auth.gr

'shafi@bartol.udel.edu
}

Published by the American Physical Society under the terms of the Creative Commons Attribution 4.0 International license. Further distribution of this work must maintain attribution to the author(s) and the published article's title, journal citation, and DOI. Funded by SCOAP. monopole-antimonopole pairs connected by magnetic $Z$-flux tubes. The magnetic dumbbell, with mass estimated to lie in the $\mathrm{TeV}$ range, is closely related, it appears, to objects of essentially the same name discussed some time ago by Nambu [10] in the standard electroweak model. However, there is an important difference between these two cases, which is related to the fact that the electroweak monopole in $S U(5)$ is associated with the induced vacuum expectation value (VEV) of a heavy $S U(2)_{L}$ triplet field with zero weak hypercharge $Y$ that resides in the adjoint Higgs 24-plet. There is, of course, no corresponding elementary $S U(2)_{L}$ triplet scalar in the standard $S U(2)_{L} \times$ $U(1)_{Y}$ model. The electroweak monopole in $S U(5)$ carries a Dirac charge of $(3 / 4)(2 \pi / e)$ as well as Z-magnetic charge, and it is expected to exist in a confined state together with its antimonopole. (Monopoles carrying Coulomb and $Z$-flux with the aim of confining primordial monopoles were previously discussed in Ref. [11]. For two basic papers on electroweak strings, see Ref. [12].)

We should emphasize that our results also apply to extensions of the minimal $S U(5)$ model that incorporate additional matter and/or scalar fields to implement gauge coupling unification and improve fermion mass relations, and are compatible with the proton lifetime limits-see, for example, Ref. [13].

It is important to clarify that the $S U(5)$ model does not predict topologically stable electroweak monopoles or strings associated with the electroweak breaking. To this end, we concentrate on the electroweak sector of the model, 
where the generator of $U(1)_{Y}$ is taken as $2 Y$ so that it has integral eigenvalues and, thus, periodicity $2 \pi$. We note that, inside $S U(5)$, the electroweak gauge symmetry is $G=S U(2)_{L} \times U(1)_{Y} / Z_{2}$, where $Z_{2}$ is generated by the element $(-1,-1)=\left(e^{i \pi T_{L}^{3}}, e^{i \pi 2 Y}\right)$ of $S U(2)_{L} \times U(1)_{Y}$ with $T_{L}^{3}=\operatorname{diag}(1,-1)$. Indeed, this element acts as the identity element on the $S U(2)_{L}$ doublet with $Y=-1 / 2$ and, consequently, on all the representations of the model including the real triplet with $Y=0$, as well as any other complex triplets with integral hypercharge. The second homotopy group of the vacuum manifold is

$$
\pi_{2}\left(\frac{G}{U(1)_{e m}}\right)=\pi_{1}\left(U(1)_{e m}\right)_{G},
$$

where the right-hand side consists of all the homotopically nontrivial loops in the electromagnetic gauge group $U(1)_{\mathrm{em}}$ which are trivial in $G$. The smallest nontrivial loop in $U(1)_{e m}$ corresponds to a $2 \pi$ rotation along the electric charge generator $Q=\left(T_{L}^{3}+2 Y\right) / 2$, which is equivalent to a $\pi$ rotation along $T_{L}^{3}$ accompanied by a $\pi$ rotation along $2 Y$ interpolating between $(1,1)$ and $(-1,-1)$ in $G$. This is the smallest closed loop in $G$ and is homotopically nontrivial. The fundamental (first homotopy) group in the right-hand side of Eq. (1) is therefore trivial, and there are no topologically stable electroweak monopoles. Moreover, the fundamental group of the vacuum manifold

$$
\pi_{1}\left(\frac{G}{U(1)_{e m}}\right)=\pi_{0}\left(U(1)_{e m}\right)_{G}
$$

is also trivial, since both $G$ and $U(1)_{e m}$ are connected, and no stable strings appear either.

In the electroweak model, we introduce a real Higgs triplet field $T=T_{i} \sigma_{i} / 2$ with a vanishing hypercharge $Y$, where $\sigma_{i}(i=1,2,3)$ are the three Pauli matrices. This triplet, which resides in the $S U(5)$ adjoint Higgs 24-plet, couples to the electroweak doublet $H$, as displayed in the following additional contribution to the potential energy density:

$$
V_{T}=\frac{1}{2} M_{T}^{2}\left(T_{i}-\frac{\lambda_{T}}{M_{T}} H^{\dagger} \sigma_{i} H\right)^{2} .
$$

Here, $M_{T} \gg M_{Z}$ is the triplet mass, which may be as large as the grand unification scale, and $\lambda_{T}$ is a dimensionless coupling constant of the order of unity or less. The cross term in the above expression originates from the Higgs couplings $5^{\dagger} \times 24 \times 5$ and $5^{\dagger} \times 24^{2} \times 5$ [14] between the $S U(5)$ Higgs 24-plet and the Higgs 5-plet which contains the electroweak doublet $H$. It yields a $T$ VEV suppressed relative to the a $\operatorname{VEV}\langle H\rangle$ of $H$ by a factor $\langle H\rangle / M_{T}$. In Eq. (3), we have left out quartic scalar couplings such as $\left(\operatorname{Tr}\left(T^{2}\right)\right)^{2}$ and $\left(H^{\dagger} H\right) \operatorname{Tr}\left(T^{2}\right)$ which do not play an essential role here.
The spontaneous breaking of the electroweak symmetry is achieved, as usual, via the potential

$$
V_{H}=\frac{\lambda}{4}\left(H^{\dagger} H-\frac{v_{D}^{2}}{2}\right)^{2},
$$

which yields the following VEV for the electroweak doublet $H$ :

$$
\langle H\rangle=\left(\begin{array}{c}
\frac{v_{D}}{\sqrt{2}} \\
0
\end{array}\right),
$$

where $v_{D} \simeq 246 \mathrm{GeV}$. From Eq. (3), this induces the triplet VEV given by

$$
\left\langle T_{3}\right\rangle=\frac{\lambda_{T} v_{D}^{2}}{2 M_{T}} \equiv v_{T},
$$

which breaks $S U(2)_{L}$ to its $U(1)_{L}$ subgroup with generator $T_{L}^{3}=\operatorname{diag}(1,-1)$.

Ignoring for the moment the electroweak symmetry breaking by $\langle H\rangle$, the breaking of $S U(2)_{L}$ by the Higgs triplet $T$ yields a 't Hooft-Polyakov-type monopole [15] with magnetic flux corresponding to a $2 \pi$ rotation around $T_{L}^{3}$ or, equivalently, a $4 \pi$ rotation around the customarily normalized generator $T_{L}^{3} / 2$. Reintroducing $\langle H\rangle$, this monopole ceases to be topologically stable and becomes attached to a magnetic flux tube. Indeed, the electroweak symmetry breaking leaves unbroken the electric charge generator $Q=T_{L}^{3} / 2+Y$, where the weak hypercharge operator is given by $Y=\operatorname{diag}(-1 / 3,-1 / 3,-1 / 3$, $1 / 2,1 / 2)$ in $S U(5)$. The corresponding orthogonal broken generator is $\mathcal{B}=T_{L}^{3} / 2-3 Y / 5$.

At this stage, it is convenient to consider $5 \mathcal{B}$, which has the smallest possible integer elements and, thus, periodicity $2 \pi$. A rotation by $2 \pi / 4$ along $5 \mathcal{B}$ leaves invariant the VEV of $H$, and, therefore, the associated tube carries $Z$-magnetic flux corresponding to a $2 \pi$ rotation around $5 \mathcal{B} / 4$. From the relation $T_{L}^{3}=3 Q / 4+5 \mathcal{B} / 4$, we see that the monopole with one unit of flux along $T_{L}^{3}$ (i.e., corresponding to a $2 \pi$ rotation around this generator) is attached to a $Z$-flux tube with one unit of flux along $5 \mathcal{B} / 4$ and also has Coulomb flux $3 Q / 4$. The magnetic charge corresponding to the Coulomb flux of the monopole is $g_{M}=(3 / 4)(2 \pi / e)$. A monopole and an antimonopole are expected to pair up and form a dumbbell connected by this flux tube.

A few remarks about the $Z$-magnetic flux emerging from the electroweak monopole are in order here. The normalized generator orthogonal to $Q$ is $\sqrt{5 / 8}\left(T_{3}^{L} / 2-3 Y / 5\right)$. As we have shown, the $Z$-magnetic flux in the tube corresponds to a $2 \pi$ rotation around $(5 / 4)\left(T_{3}^{L} / 2-3 Y / 5\right)$. Consequently, this flux is $(2 \pi / g) \sqrt{5 / 2}$, where $g$ is the 
$S U(5)$ gauge coupling which, in the $S U(5)$ limit, coincides with the $S U(2)_{L}$ gauge coupling $g=e / \sqrt{3 / 8}$. The magnetic flux along the $Z$ tube therefore takes the form $(4 \pi / e) \sqrt{3 / 8} \sqrt{5 / 8}$. For completeness, we should note that the expressions above for the Coulomb and $Z$-magnetic fluxes of the electroweak monopole coincide, respectively, with the values $4 \pi \sin ^{2} \theta_{W} / e$ and $4 \pi \sin \theta_{W} \cos \theta_{W} / e$ found by Nambu [10] by recalling the $S U(5)$ prediction $\sin ^{2} \theta_{W}=3 / 8$, where $\theta_{W}$ is the electroweak angle. Combining appropriately these two fluxes, one obtains the total $S U(2)_{L}$ magnetic charge $4 \pi / g$ of the electroweak monopole, in full agreement with the results found by Nambu [10] and Vachaspati [16].

To reconfirm that the electroweak monopole accompanied by a $Z$-magnetic flux tube provides a consistent description, let us take the left-handed neutrino with zero electric charge around this tube. If the neutrino is covariantly transported, its wave function acquires an AharonovBohm phase given by $\exp \left(i Q_{Z}^{\nu} \Phi_{Z}\right)$, where $Q_{Z}^{\nu}$ is the neutrino $Z$ charge and $\Phi_{Z}$ denotes the $Z$-magnetic flux in the tube. Substituting $Q_{Z}^{\nu}=\left(e / \sin \theta_{W} \cos \theta_{W}\right)\left(T_{L}^{3} / 2-\right.$ $\left.Q \sin ^{2} \theta_{W}\right)$ with $T_{L}^{3}=+1, Q=0$, and requiring the wave function to be single valued shows that the $Z$-magnetic flux $\Phi_{Z}$ is quantized in units of $(4 \pi / e) \sin \theta_{W} \cos \theta_{W}$, which is in agreement with the discussion above. A related calculation for the charged leptons and quarks also takes into account the ordinary Coulomb magnetic flux which is carried by the electroweak monopole. As an example, for the left-handed d quark kept within its confinement radius $\sim \Lambda_{\mathrm{QCD}}^{-1}\left(\gg M_{Z}^{-1}\right)$, the total phase acquired by its wave function, taking into account the $Z$ flux and Coulomb flux $\Phi_{e m}$, is given by $\left(e / \sin \theta_{W} \cos \theta_{W}\right)\left(T_{L}^{3} / 2-Q \sin ^{2} \theta_{W}\right) \Phi_{Z}+e Q \Phi_{e m}$, with $T_{L}^{3}=-1$ and $Q=-1 / 3$. Substituting the $Z$ flux $\Phi_{Z}=$ $4 \pi \sin \theta_{W} \cos \theta_{W} / e$ and requiring the wave function to be single valued yields $\Phi_{e m}=(4 \pi / e) \sin ^{2} \theta_{W}$ as a solution. This is the desired value of the Coulomb magnetic flux which appropriately combined with the $Z$ flux of the monopole yields the $S U(2)_{L}$ magnetic flux of $(4 \pi / e) \sin \theta_{W}$. Note that the wave function of an $S U(2)_{L}$ singlet quark or lepton acquires a zero overall phase under similar transport. This is consistent with the fact that the electroweak monopole is associated with the breaking of $S U(2)_{L}$ to $U(1)_{L}$.

In the above discussion which is based on $S U(2)_{L} \times$ $U(1)_{Y}$, the value of the electroweak mixing angle $\theta_{W}$ is not predicted. The situation in grand unified theories, however, is different, and the prediction $\sin ^{2} \theta_{W}=3 / 8$ allows us to provide the magnitude of the $Z$-magnetic flux.

Finally, the quark confinement that we assumed above is not required for consistency in the presence of the electroweak monopole that also carries a $Z$-magnetic flux tube. However, we recall from the introduction that $S U(5)$ also predicts the existence of a topologically stable monopole that carries a Dirac charge of $2 \pi / e$ as well as a screened color magnetic field. Quark confinement in this case is required in order for the Dirac quantization condition to be satisfied beyond the screening radius $\sim \Lambda_{\mathrm{QCD}}^{-1}$.

Returning to Eqs. (3) and (4), we should note that, at tree level, the VEV of $H$ is not affected by the presence of the Higgs triplet $T$. Indeed, minimization of the combined potential $V=V_{H}+V_{T}$ is achieved at

$$
M_{T}^{2}\left(T_{i}-\frac{\lambda_{T}}{M_{T}} H^{\dagger} \sigma_{i} H\right)=0,
$$

and

$$
\begin{gathered}
-M_{T}^{2}\left(T_{i}-\frac{\lambda_{T}}{M_{T}} H^{\dagger} \sigma_{i} H\right) \frac{\lambda_{T}}{M_{T}} H^{\dagger} \sigma_{i} \\
+\frac{\lambda}{2}\left(H^{\dagger} H-v_{D}^{2}\right) H^{\dagger}=0 .
\end{gathered}
$$

In view of Eq. (7), Eq. (8) reduces to the standard equation for the electroweak symmetry breaking:

$$
\frac{\lambda}{2}\left(H^{\dagger} H-\frac{v_{D}^{2}}{2}\right)=0,
$$

and so the presence of the triplet $T_{i}$ does not affect the VEV of the doublet $H$.

The $\rho$ parameter [17] in our case is given by $\rho=$ $1+4 R^{2}$, with $R=v_{T} / v_{D}$ (see, e.g., Ref. [18]). From the $2 \sigma$ upper bound $\rho \lesssim 1.00077$ [19], we find $v_{T} \lesssim 3.4 \mathrm{GeV}$. Equation (6) then implies that

$$
M_{T} \gtrsim 4.4\left(\frac{\lambda_{T}}{0.5}\right) \mathrm{TeV} .
$$

A triplet with mass in the $\mathrm{TeV}$ range could provide a new source for Higgs production at high-energy colliders. Moreover, the mixed quartic coupling involving $H$ and $T$ may be helpful in preventing the quartic Higgs coupling going to zero and, thereby, stabilize the electroweak vacuum. It would be interesting to explore these possibilities in more realistic models which, among other things, also implement gauge coupling unification.

With the ansatz $T_{i}=v_{T} x_{i} / r$, where $x_{i}(i=1,2,3)$ are the spatial coordinates and $r$ is the radial distance, the potential in Eq. (3) is minimized for

$$
H^{\dagger} \sigma_{i} H=\frac{v_{D}^{2}}{2} \frac{x_{i}}{r} \text {. }
$$

This is achieved by taking [10]

$$
H=\frac{v_{D}}{\sqrt{2}}\left(\begin{array}{c}
\cos \frac{\theta}{2} \\
\sin \frac{\theta}{2} e^{i \varphi}
\end{array}\right),
$$

where $0 \leq \theta<\pi$ and $0 \leq \varphi<2 \pi$ are the polar angles. It is important to note that the formula in Eq. (12) has an 
ill-defined phase $\varphi$ on the negative $x_{3}$ axis where $\theta=\pi$. This reflects the fact that the monopole is accompanied by a string ( $Z$-flux tube). (For a discussion of the stability of this string, see Ref. [20] and papers listed therein. A careful analysis is required to assess the string stability in the presence of the scalar triplet. In the discussion above, we have seen that the $Z$-flux tube was required by the Dirac quantization condition. This leads us to conjecture that the string is susceptible to breaking through a monopoleantimonopole pair creation.)

To obtain a rough estimate of the monopole mass, following Ref. [10], we ignore for the moment the attached $Z$ tube and approximate the monopole by a sphere of radius $r$ within which the gauge fields, $H$, and $T$ are zero. (Being a heavy scalar field, we expect $T$ to approach its VEV inside an inner core of radius $M_{T}^{-1}$. The energy stored in this inner core can be ignored, which can be checked by examining the quartic and gradient terms for the triplet $T$.) Outside the sphere, all the Higgs fields lie in the vacuum, and we have a Coulomb magnetic field corresponding to the magnetic charge $[10,16,21,22]$

$$
g_{M}=\frac{4 \pi}{e} \sin ^{2} \theta_{W}
$$

where $e$ is the absolute value of the electron charge. The energy of the monopole configuration is then

$$
E_{M}=\frac{g_{M}^{2}}{8 \pi r}+\frac{4 \pi}{3} r^{3} V_{0}
$$

where $V_{0}$, the potential energy density within the sphere, is given by

$$
V_{0}=\frac{\lambda v_{D}^{4}}{16}=\frac{m_{H}^{2} v_{D}^{2}}{8}
$$

and $m_{H}=\sqrt{\lambda / 2} v_{D}$ is the Higgs boson mass. The energy $E_{M}$ is minimized at

$$
\begin{aligned}
r_{\min } & =\left(\frac{g_{M}^{2}}{32 \pi^{2} V_{0}}\right)^{1 / 4}=\sqrt{\frac{2}{e}} \sin \theta_{W}\left(m_{H} v_{D}\right)^{-1 / 2} \\
& \simeq 7 \times 10^{-3} \mathrm{GeV}^{-1},
\end{aligned}
$$

giving the monopole mass

$$
\begin{aligned}
m_{M} & \approx \frac{2^{1 / 4} g_{M}^{3 / 2} V_{0}^{1 / 4}}{3 \pi^{1 / 2}}=\frac{2^{5 / 2} \pi \sin ^{3} \theta_{W}\left(m_{H} v_{D}\right)^{1 / 2}}{3 e^{3 / 2}} \\
& \simeq 688 \mathrm{GeV} .
\end{aligned}
$$

One can calculate the $Z$-tube radius $\rho_{\text {str }}$ and tension $\mu_{\text {str }}$ following Ref. [10]. We find $\rho_{\mathrm{str}} \simeq 1.86 \times 10^{-2} \mathrm{GeV}^{-1} \quad$ and $\quad \mu_{\mathrm{str}} \simeq 2.57 \times 10^{5} \mathrm{GeV}^{2}$

The string radius exceeds the monopole radius by a factor of 2.5 or so. So it makes sense to consider a string segment at least as long as its radius. The energy of the "minimal" string segment is about $4.8 \mathrm{TeV}$, which yields a minimal dumbbell of $5.8 \mathrm{TeV}$, after including the potential energy from the Coulomb attraction between the monopoleantimonopole pair.

Nambu has argued [10] that a rotating relativistic dumbbell with energy $E$ and angular momentum $L$ may yield a Regge trajectory $L \sim \alpha_{0}^{\prime} E^{2}$, with $\alpha_{0}^{\prime}=1 /\left(2 \pi \mu_{\text {str }}\right)$. Using the relevant formulas in Ref. [10], we find that, for string lengths bigger than the minimal length, $L \gtrsim 35$ and $E \gtrsim 7.5 \mathrm{TeV}$. The dumbbell is expected to decay through the emission of photons, weak gauge bosons, hadrons, and leptons with lifetime $(E / 6.74) \times 10^{-5} \mathrm{GeV}^{-1} \gtrsim 7.33 \times$ $10^{-27} \mathrm{sec}$ [10]. The string can also decay by monopole pair creation with the decay rate per unit length [23]

$$
\Gamma_{\mathrm{str}}=\frac{\mu_{\mathrm{str}}}{2 \pi} e^{-\pi \frac{m_{M}^{2}}{\mu_{\mathrm{str}}}} \simeq 127 \mathrm{GeV}^{2} .
$$

The corresponding lifetime for a dumbbell with energy $E$ is $(E / 5.13) \times 10^{-7} \mathrm{GeV}^{-1} \gtrsim 9.64 \times 10^{-29} \mathrm{sec}$, which is shorter than its radiative lifetime by about 2 orders of magnitude. (For a discussion on the evolution of dumbbells, see Ref. [24].)

With the electroweak monopole mass estimated to be around $700 \mathrm{GeV}$, it is plausible that highly unstable configurations, consisting of (overlapping) monopoleantimonopole pairs in a mass range of 1-2 TeV, may be produced in high-energy collisions.

Although we have discussed the presence of the electroweak monopole and $\mathrm{TeV}$-scale magnetic dumbbells in minimal $S U(5)$, one could reasonably expect that similar structures also appear in larger grand unified theories. Indeed, based on our discussion, they seem to be a rather generic feature of such theories. Consider $S O(10)$, for instance, in which a nonzero VEV for the $S U(2)_{L}$ triplet $T$ will arise through the mixed quartic coupling $45^{2} \times$ $10 \times 10$. Here, the 10-plet contains the standard Higgs doublet, and the 45-plet is the adjoint Higgs field. In a more elaborate $S O(10) \times U(1)_{P Q}$ model [25], where $U(1)_{P Q}$ denotes the axion symmetry [26], the trilinear mixed coupling $10 \times 10 \times 45$ will induce a nonzero VEV for the $S U(2)_{L}$ triplet in the 45-plet. The presence of TeV-scale magnetic dumbbells, made up of monopole-antimonopole pairs, thus appears to be a rather generic feature of grand unified theories. Furthermore, the $S O(10)$ breaking to the standard model often proceeds through one or more intermediate steps. Suppose that the low-energy group, excluding QCD, is $S U(2)_{L} \times S U(2)_{R} \times U(1)_{B-L}$. In this 
case, we could proceed to break $S U(2)_{R}$ to $U(1)_{R}$ with an $S U(2)_{R}$ triplet scalar, which yields a monopole carrying $U(1)_{R}$ charge. With the next breaking of $U(1)_{R} \times U(1)_{B-L}$ to $U(1)_{Y}$, the monopole gets connected to a string (flux tube). We, therefore, expect the appearance of new dumbbells, and, if the monopole mass scale for this case is suitably large compared to the string scale, the flux tubes can be relatively stable and less likely to break via monopole-antimonopole pair creation. The dumbbell mass may lie in the $\mathrm{TeV}$ range depending on the symmetrybreaking scale of $S U(2)_{L} \times S U(2)_{R} \times U(1)_{B-L}$.

Let us mention that our discussion carries over to the supersymmetric extensions of the $S U(5)$ and $S O(10)$ models. The induced VEV for the scalar triplet $T$ arises in supersymmetric $S U(5)$, for instance, via the soft supersymmetry-breaking terms corresponding to the superpotential coupling $\overline{5} \times 24 \times 5$, where $\overline{5}$ and 5 contain the two Higgs doublets. Consequently, this VEV is suppressed by an additional factor $m_{0} / M_{T}$, where $m_{0}$ denotes the soft supersymmetry-breaking mass parameter. The electroweak monopole is therefore also expected to be present in these models.

In summary, we have identified in $S U(5)$ the presence of an electroweak monopole that carries a Dirac magnetic charge of $(3 / 4)(2 \pi / e)$ and a $Z$-magnetic flux. Under plausible assumptions, the monopole mass is estimated to be around $700 \mathrm{GeV}$, and the associated $Z$-flux tube width and tension are of the order of $M_{Z}^{-1}$ and $30 M_{Z}^{2}$, respectively. The monopole-antimonopole pairs form dumbbells (mesons) discovered some time ago by Nambu. A search for these extended structures at the LHC and its upgrades seems worthy of further consideration. Finally, we have noted that analogous $\mathrm{TeV}$-scale extended structures can also appear in larger gauge symmetries such $S O(10)$.

\section{ACKNOWLEDGMENTS}

This work is supported by the Hellenic Foundation for Research and Innovation (H. F. R. I.) under the "First Call for H.F.R.I. Research Projects to support Faculty Members and Researchers and the procurement of highcost research equipment grant" (Project No. 2251). Q. S. is supported in part by the Department of Energy Grant No. DE-SC-001380.

Note added in proof.-For another discussion of the electroweak monopole with different conclusions, see Ref. [27].
[1] H. Georgi and S. L. Glashow, Phys. Rev. Lett. 32, 438 (1974).

[2] H. Georgi, AIP Conf. Proc. 23, 575 (1975); H. Fritzsch and P. Minkowski, Ann. Phys. (N.Y.) 93, 193 (1975).

[3] F. Gürsey, P. Ramond, and P. Sikivie, Phys. Lett. 60B, 177 (1976); Y. Achiman and B. Stech, Phys. Lett. 77B, 389 (1978); Q. Shafi, Phys. Lett. 79B, 301 (1978).

[4] P. A. M. Dirac, Proc. R. Soc. A 133, 60 (1931).

[5] C. P. Dokos and T. N. Tomaras, Phys. Rev. D 21, 2940 (1980).

[6] M. Daniel, G. Lazarides, and Q. Shafi, Nucl. Phys. B170, 156 (1980); G. Lazarides, Q. Shafi, and W. P. Trower, Phys. Rev. Lett. 49, 1756 (1982).

[7] J. C. Pati and A. Salam, Phys. Rev. D 10, 275 (1974); 11, 703(E) (1975).

[8] G. Lazarides, M. Magg, and Q. Shafi, Phys. Lett. 97B, 87 (1980).

[9] G. Lazarides and Q. Shafi, arXiv:2101.01412.

[10] Y. Nambu, Phys. Rev. D 10, 4262 (1974); Nucl. Phys. B130, 505 (1977).

[11] G. Lazarides and Q. Shafi, Phys. Lett. 94B, 149 (1980).

[12] T. Vachaspati, Phys. Rev. Lett. 68, 1977 (1992); 69, 216(E) (1992); Nucl. Phys. B397, 648 (1993).

[13] M. U. Rehman, Q. Shafi, and J. R. Wickman, Phys. Rev. D 78, 123516 (2008).

[14] A. J. Buras, J. R. Ellis, M. K. Gaillard, and D. V. Nanopoulos, Nucl. Phys. B135, 66 (1978).
[15] G. 't Hooft, Nucl. Phys. B79, 276 (1974); A. M. Polyakov, JETP Lett. 20, 194 (1974).

[16] T. Vachaspati, Nucl. Phys. B439, 79 (1995).

[17] M. J. G. Veltman, Phys. Lett. 91B, 95 (1980).

[18] J. L. Díaz-Cruz and D. A. López-Falcón, Phys. Lett. B 568, 245 (2003).

[19] M. Tanabashi et al., Phys. Rev. D 98, 030001 (2018).

[20] M. James, L. Perivolaropoulos, and T. Vachaspati, Phys. Rev. D 46, R5232 (1992); Nucl. Phys. B395, 534 (1993).

[21] A. Achucarro and T. Vachaspati, Phys. Rep. 327, 347 (2000).

[22] J. Preskill, Phys. Rev. D 46, 4218 (1992).

[23] A. Monin and M. B. Voloshin, Phys. Rev. D 78, 065048 (2008); L. Leblond, B. Shlaer, and X. Siemens, Phys. Rev. D 79, 123519 (2009); A. Monin and M. B. Voloshin, Phys. At. Nucl. 73, 703 (2010); W. Buchmuller, V. Domcke, and K. Schmitz, Phys. Lett. B 811, 135914 (2020).

[24] J. Urrestilla, A. Achúcarro, J. Borrill, and A. R. Liddle, J. High Energy Phys. 08 (2002) 033.

[25] R. Holman, G. Lazarides, and Q. Shafi, Phys. Rev. D 27, 995 (1983); G. Lazarides and Q. Shafi, Phys. Lett. B 807, 135603 (2020).

[26] R. D. Peccei and H. R. Quinn, Phys. Rev. Lett. 38, 1440 (1977); S. Weinberg, Phys. Rev. Lett. 40, 223 (1978); F. Wilczek, Phys. Rev. Lett. 40, 279 (1978).

[27] P. Q. Hung, Nucl. Phys. B962, 115278 (2021); J. Ellis, P. Q. Hung, and N. Mavromatos, arXiv:2008.00464. 